\title{
LOCATION BASED SERVICE IN INDOOR ENVIRONMENT USING QUICK RESPONSE CODE TECHNOLOGY
}

\author{
Farshad Hakimpour $^{\mathrm{a},{ }^{*}}$, Ali Zare Zardiny ${ }^{\mathrm{a}}$ \\ a Department of Geomatics Engineering, Faculty of Engineering, University of Tehran, (fhakimpour, zare_zardiny)@ut.ac.ir
}

KEYWORDS: Location Based Service, QR Code, Indoor Positioning, NFC, RFID, WiFi

\begin{abstract}
:
Today by extensive use of intelligent mobile phones, increased size of screens and enriching the mobile phones by Global Positioning System (GPS) technology use of location based services have been considered by public users more than ever.. Based on the position of users, they can receive the desired information from different LBS providers. Any LBS system generally includes five main parts: mobile devices, communication network, positioning system, service provider and data provider. By now many advances have been gained in relation to any of these parts; however the users positioning especially in indoor environments is propounded as an essential and critical issue in LBS. It is well known that GPS performs too poorly inside buildings to provide usable indoor positioning. On the other hand, current indoor positioning technologies such as using RFID or WiFi network need different hardware and software infrastructures. In this paper, we propose a new method to overcome these challenges. This method is using the Quick Response (QR) Code Technology. QR Code is a 2D encrypted barcode with a matrix structure which consists of black modules arranged in a square grid. Scanning and data retrieving process from QR Code is possible by use of different camera-enabled mobile phones only by installing the barcode reader software. This paper reviews the capabilities of QR Code technology and then discusses the advantages of using QR Code in Indoor LBS (ILBS) system in comparison to other technologies. Finally, some prospects of using QR Code are illustrated through implementation of a scenario. The most important advantages of using this new technology in ILBS are easy implementation, spending less expenses, quick data retrieval, possibility of printing the QR Code on different products and no need for complicated hardware and software infrastructures.
\end{abstract}

\section{Introduction}

Today by spread of using mobile phones, increased the size and power and mobile phones being equipped with GPS technology, use of LBS have huge growth and significant development. LBS systems integrate the location of a mobile phone with other information to provide different types of services and useful information to a mobile user. Any LBS system needs different infrastructure components to work. These components are: mobile devices, communication network, positioning system, application server and data provider. As mentioned, LBS systems provide different services based on the user position; accordingly, ability to determine user position is an essential part of any LBS system. Position and location data can be derived from different kinds of sources. One of the most common and very popular positioning technologies is GPS but it is well known that GPS performs too poorly inside buildings to provide usable indoor positioning, since the required satellite links are blocked or unreliable inside buildings. It is for that reason that indoor positioning systems are considered much more challenging than outdoor. With regards to weakness of GPS, many different indoor positioning systems have been developed by now (e.g. WiFi network, RFID or NFC) that each needs different hardware and software infrastructures.

In the past years several researches was done in the field of ILBS. Johannes Bolz (2011) used NFC technology for linking location and information associated with it. He developed a demonstrator application running on the Android OS that could get the location information by reading NFC tags. Eladio Martin et.al. (2010) developed an application for indoor localization using WiFi signal strength. Muhammad Usman (2012), designed and implemented a prototype smart phone application that is capable of indoor and outdoor LBSs called “Combined Indoor Outdoor Navigator” using different technologies such as GPS, WiFi and QR Code. Joe Murphy (2012) explore the implications of the location-sensitive tools for information engagement and thus for libraries using QR Code. Indoor positioning is the part of Indoor LBS that is considered in most of these researches.

The main goal of this paper is feasibility study of using QR Code technology for different ILBS in addition to indoor positioning. In this research we design a location based tourist guide system using QR Code technology. Defined areas such as museums, galleries or exhibition centers have the opportunity to present their collections with this system. The remainder of this paper consists of five sections. Next section introduces the specification of QR Code technology. In section three, the most commonly used technology for indoor positioning (i.e. WiFi Fingerprint, RFID and NFC) are reviewed. Section four evaluates the capabilities of QR Code in comparison to other technologies being used in ILBS. In section five some prospects of using QRCode are illustrated through implementation of scenario. Finally, in Section six, the results and recommendations of paper are presented.

\section{Quick Response (QR) Code}

QR Code is a 2D encrypted barcode with a matrix structure which consists of black modules arranged in a square grid on a white background (T.J. Soon, 2008). This barcode can serve as a mobile bridge between physical platforms and digital information (Joe Murphy, 2012). In other word, QR codes are physical barcodes that, 
when scanned with a mobile application, can connect users to digital content. Scanning and data retrieving process from QR Code is possible by use of different types of mobile phones equipped with a camera. Once the appropriate scanner software is installed on the mobile device, users only require taking a snapshot of the code to decode it. QR Code is made based on standard patterns. This pattern code is shown in figure1.

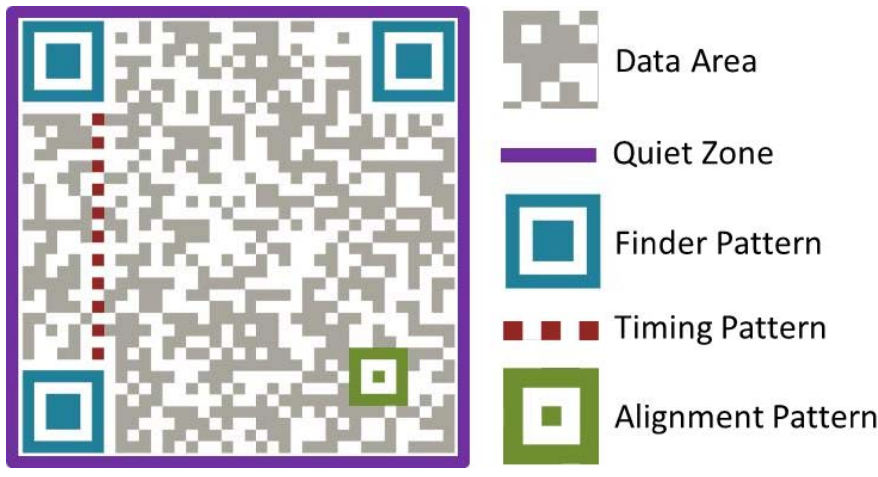

Figure 1. QR Code pattern

The elements contained in a QR code are the following:

- Finder Pattern: A pattern for detecting the position of the QR Code. Three big squares in the corners used for detecting the position, the size and the angle of the QR Code.

- Alignment Pattern: A pattern used for correcting the distortion of the QR Code. These distortions could occur for example when attaching the codes onto a curved surface.

- Timing Pattern: It consists in white and black modules arranged alternately and placed between two position patterns. It is used to determine the central coordinate of each cell in the QR Code.

- $\quad$ Quiet Zone: A margin space necessary for reading the QR Code. This margin space makes it easier to detect the QR Code. At least four cells are required for the quiet zone.

- Data Area: The area in the QR Code that contains the data encoded in binary numbers of ' 0 ' and ' 1 ' based on the encoding rule.

The information encoded by a QR code may be made up of four standardized types of data (i.e. numeric, alphanumeric, byte/binary, $\mathrm{Kanji}^{1}$ ). The amount of data that can be stored in the QR code symbol depends on the data type (T.J. Soon, 2008). The typical barcode holds a maximum of 20 digits, while the maximum data capacities of a QR code are 7,089 characters for numeric data, 4,296 characters for alphanumeric data, 2,953 bytes for binary data, and 1,817 characters for Japanese Kanji data (M.V. Briseno, 2012). In addition, QR code includes error correction. Thanks to this capability it is possible to read the barcode even if it presents some distortions or damage. There are four levels of error correction. The lowest is L, which allows the code to be read even if $7 \%$ of it is unreadable. After that is $\mathrm{M}$ whit $15 \%$, Q whit $25 \%$ and $\mathrm{H}$ whit $30 \%$ error correction (T.J. Soon, 2008).

\section{Current Indoor Positioning systems}

In this section the most commonly used technologies in indoor positioning are reviewed.

\subsection{RFID (Radio Frequency Identification)}

${ }^{1}$ A system of Japanese writing using Chinese characters
RFID as a type of auto identification system that uses radio waves to store and retrieve data from an identification chip. These chips are known as RFID tags that can affix to physical objects and carry identifying data. Usually an RFID system requires four main components: RFID reader/writer, RFID tag, application software for processing the information and databases. The reader periodically transmits signals to search for tags in their vicinity. When it captures a signal from a tag, it extracts the information and passes the data to the processing subsystem. RFID systems do not require line of sight and data can be transferred without contact between the reader and the tag. There are several methods of identifying objects using RFID, but the most common is to store a serial number that identifies an object, and perhaps other information, on a microchip that is attached to an antenna (L. McCathie, 2004). RFID systems only allow relatively low volumes of data to be stored on the tags from 8kbyte to $128 \mathrm{kbyte}$ and support read speed rate from $200 \mathrm{bits} / \mathrm{s}$ to $28 \mathrm{Kbits} / \mathrm{s}$ depending on the frequency used and type of tag (M.V. Briseno, 2012).

\subsection{Near field communication (NFC)}

NFC is a short range radio technology based on and extends on RFID system that enables communication between devices that either touch or are momentarily held close together. NFC is an open platform technology, which is being standardized in the NFC Forum. Like RFID, any NFC system contains a reader/writer and transponders (Tag). Figure 2 display structure as well as sample of NFC tags. In order to read a tag the users almost touches it with an NFC-enabled device. The maximum distance over which data can be transmitted is intended to be no more than $10 \mathrm{~cm}$ (R. Gomes, 2007).

The NFC standard has been adopted by a number of mobile phone providers in mobile phones. A NFC-enabled phone when tapped against a NFC tag can receive information such as URL or phone number or some text based upon on which an action can be defined (R. Gomes, 2007). NFC standard supports different internal memory capacity from 96 byte up to $1 \mathrm{MB}$ and different data transmission rates from $106 \mathrm{Kbits} / \mathrm{s}$ to $424 \mathrm{Kbits} / \mathrm{s}$ depending on its model (M.V. Briseno, 2012).

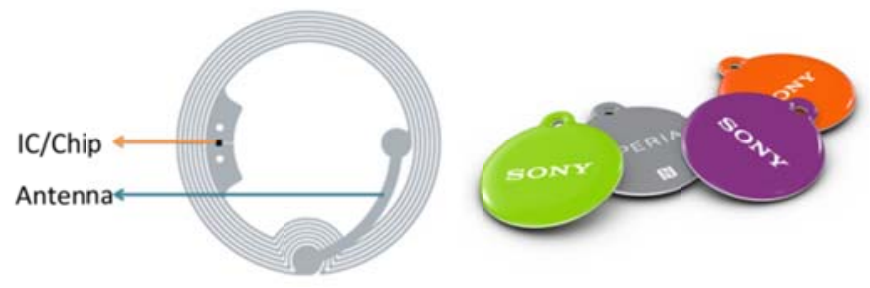

Figure 2. Strauture and sample of NFC tags

\subsection{WiFi}

Another technology for indoor positioning is using WiFi Network. The WiFi system based on IEEE 802.11 standards, works in the 2.4, 3.6 and $5 \mathrm{GHz}$ frequency bands. In WiFi networks based on strength of received signal from Access Points, we can locate mobile device inside a building. This process consists of two phase: offline and online. In offline phase, also called training phase, signal strength patterns of the area is built. In this phase measured signal strength from different APs are recorded at different locations. Each measurement consists of several readings, one for each radio source in range (Eladio Martin et.al, 2010). In online phase (positioning phase), mobile terminal infers its location through best matching between the fingerprinting signal strength being received and those previously recorded in offline phase. Localization algorithms employed in this 
case generally make use of deterministic or probabilistic techniques (Eladio Martin et.al, 2010).

\section{Comparison of the technologies}

In previous section, we describe the most common used technologies in indoor positioning. In this section we evaluate the capabilities of QR Code in comparison to other technologies to be used in ILBS system. Therefor we consider several items that are important in ILBS system and compare these technologies based on these items. These items are:

- Availability in mobile phone: QR Code reading apps are available for almost any camera-enabled mobile phone by different platform (e.g. iPhone, Android, Windows Mobile and JavaME) while at the moment the number of NFC-enabled and RFID-enabled mobile phones is still very limited.

- $\quad$ Cost of generating the barcode/tag: QR Code tags can be easily generated using free online generators and can be printed with any ordinary printer using common paper, while NFC and RFID tags require special devices generate them. Therefore these tags are more expensive than the QR Code tags.

- Internal memory capacity: QR Code in comparison to RFID or NFC tag has more internal memory capacity. This volume is 2,953 byte while NFC and RFID tag have respectively maximum $1 \mathrm{MB}$ and 28kbyte internal memory.

- Cost and time of setup: All current approaches to indoor positioning require a technological consideration and infrastructure that must be implemented in the respective building. For example software and support personnel needed to install and operate the RFID reading systems may be more costly (McCathie, 2004). While Positioning with QR Code in comparison to other technologies does not require additional hardware installments that reduce total system cost as well as setup time.

- Need to preprocessing: As mentioned in previous section location fingerprinting need an offline phase to generate the signal strength patterns and users are located by performing the probabilistic (best matching) process on this pattern while positioning by QR Code does not require any process.

- Interfere: One of the challenges in using WiFi, RFID and NFC is problem of readers discriminating between signals from multiple transponders and APs while in QRCode there is no concern about this problem.

- Vulnerable to damage: Because the electrical structure of RFID or NFC tags, these tags cannot be read well when placed on metal or liquid objects or when these objects are between the reader and the tag. Also environmental considerations including chemical, vibration, shock, moisture, electrical interference, static discharge or high powered magnetic surges may damage the tags. But QR Code can be printed on plain paper and attached to any object. QR Codes can also be delivered digitally, on the internet or television.

- Data Restoration Functionality: Thank to error correction functionality in QR Code, these barcodes can be read correctly even when they are smudged or damaged up until the error correction level while if wires in RFID and NFC tags are damaged, the tag cannot be read.

With regard to the capabilities of QRCode in comparison to other technologies, we proposed the use of QR Code for LBS system in indoor environment. Use of QRCode in ILBS not only does not require highly complicated software and hardware infrastructure, but it also reduces the time and cost of system implementation and setup.

\section{Implementation}

In this section, for illustration of some prospects of using QR Code, we designed a location based tourist guide system in indoor environment. Defined areas such as museums, galleries or exhibition centers have the opportunity to present their collections with this system. For implementating this scenario we used two types of QR Code that are Positioning and Identifier Barcodes. For user positioning inside the building, we considered QR Code barcodes as control points (points with certain coordinates). Positioning barcodes are installed on different places of building. Any QR Code includes a Geo URI that consists of geo coordinates of each location where they would be installed and address of providing server. When the user scans the barcodes with his/her mobile phone, a request is sent to providing service. Response of this request is a map that displays user position inside the building. Like any LBS system, implemented system consists of several components. Figure 3 displays these components.

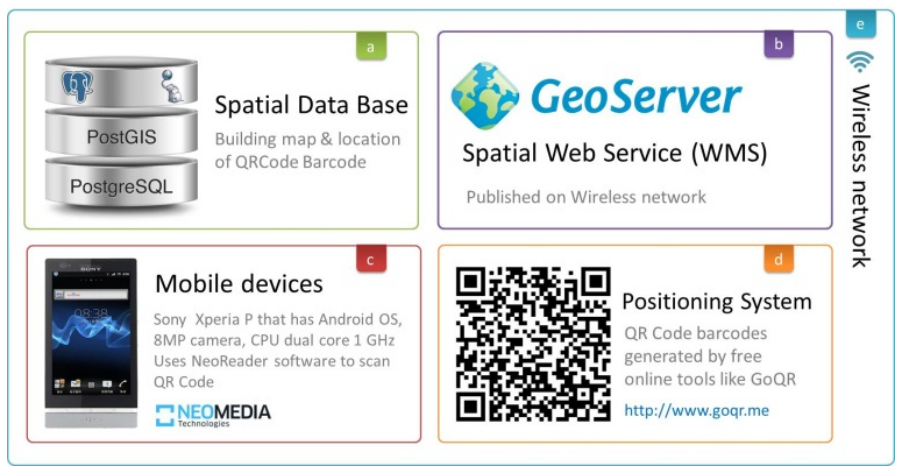

Figure 3. Component of implemented LBS system (a.Data Provider, b.Service Provider, c.Mobile Device, d.Positioning System, e.Communication Network)

In this scenario, Web Map Service receives the user request through the wireless network and based on this request retrieves spatial data from database and delivers them in a png or jpg image. To display the output on mobile Web browser we used OpenLayer software (Figure 4)

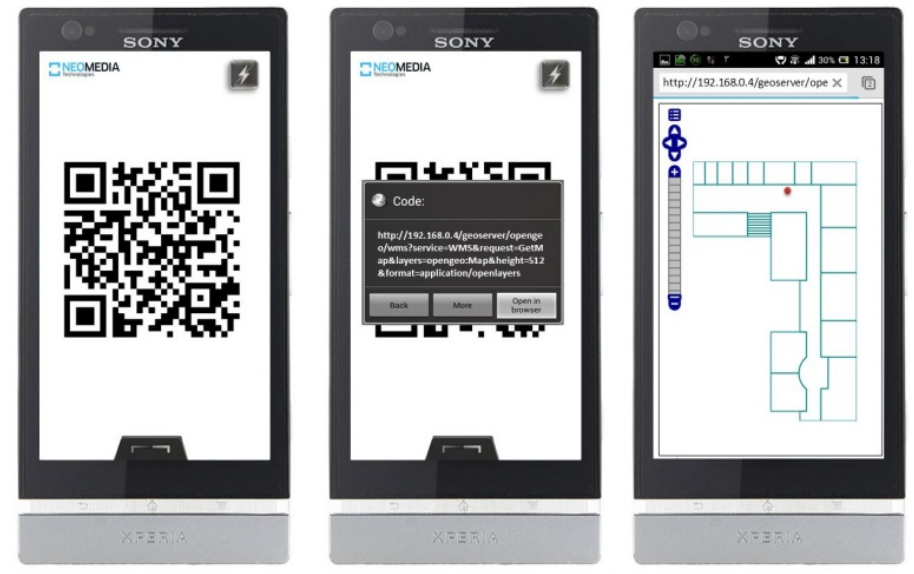

Figure 4. The map presented each marker's position as a red dot

Second types of barcodes (i.e. identifier barcodes) are used for implementation of an Object Hyperlink system. Object hyperlinking is a new term that extends the current Internet to the real world (M.V. 
Briseno, 2012). This is done by attaching identifier tags to the different objects that are in a museums or a gallery. These tags contain useful information related to object (e.g. pictures, Web pages, voices or videos) that can be retrieved and displayed by a mobile device. For example when a tourist visits from a museum, he/she can access to desire information by using the QR Codes.
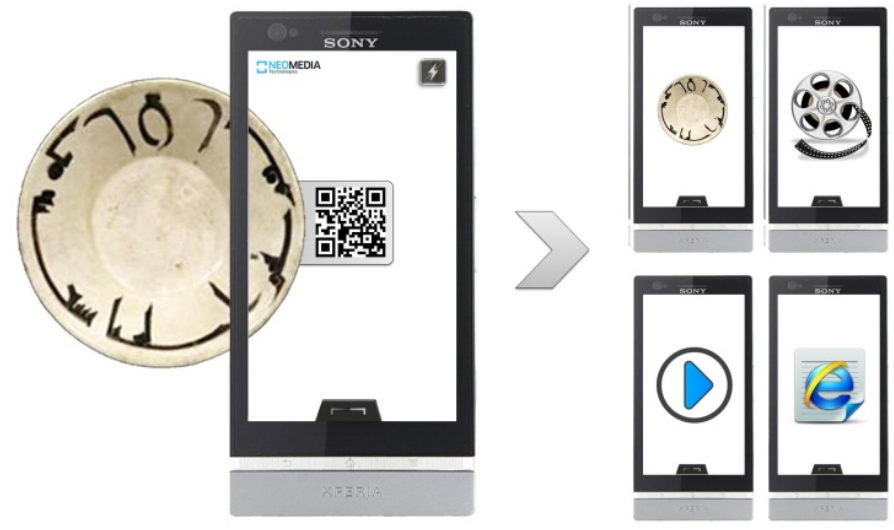

Figure 5. Identifier QR Code contains a URL address of a web page, pictures, voice or video related to object.

\section{Conclusion and Future Work}

In this paper the idea of using QRCode technology is proposed, in order to improve the Indoor Location Based Services. Also current technologies for indoor positioning are discussed. Then we evaluate capabilities of QRCode technology in comparison to current technology (WiFi, RFID and NFC). The most important advantages of using this new technology in indoor LBS systems are easy implementation, spending less expenses, quick data retrieving, possibility of scanning barcode from different rotating view, no need to preprocessing, more internal memory capacity, possibility of printing the QR Code on different products and no need for complicated hardware and software infrastructures. In implementation section we design a location based tourist guide system in indoor environment using QR Code technology.

\section{References}

Johannes Bolz, 2011, Indoor Positioning using NFC Tags, Bachelor Thesis, Berlin

Eladio Martin, Oriol Vinyals, Gerald Friedland, Ruzena Bajcsy, 2010, Precise Indoor Localization Using Smart Phones, University of. California, Berkeley. Berkeley, CA

Muhammad Usman, February 2012, Design and Implementation of an iPad Web Application for Indoor-Outdoor Navigation and Tracking Locations, Master's Thesis, Department of Surveying and Planning, School of Engineering, Aalto University, Espoo

Tan Jin Soon, 2008, QR Code, synthesis journal, section three, Automatic Data Capture Technical Committee

Mabel Vazquez Briseno, Francisco I. Hirata, Juan de Dios Sanchez Lopez, Elitania Jimenez-Garcia, Christian Navarro Cota and Juan Ivan Nieto Hipolito, 2012, Using RFID/NFC and QR Code in Mobile Phones to Link the Physical and the Digital World, Autonomous University of Baja California, Mexico
L. McCathie, 2004, The advantages and disadvantages of barcodes and radio frequency identification in supply chain management, Faculty of Informatics, Honours Theses (Archive) University of Wollongong Thesis Collections

Joe Murphy, 2012, Location Aware Services and QR Codes for Libraries, Neal-Schuman Publishers, chapter 1, pp 3-5

Rodolfo Gomes, 2007, Introduction to NFC (Near Field Communication), $16^{\text {th }}$ IST Mobile \& Wireless Communication Summit, Budapest, Hungary 